\title{
Fibromyalgia and arthritides
}

\author{
F. Atzeni ${ }^{1}$, S. Salli ${ }^{2}$, M. Benucci ${ }^{3}$, M. Di Franco ${ }^{4}$, \\ R. Casale ${ }^{5}$, A. Alciati $^{6}$, P. Sarzi-Puttini ${ }^{1}$ \\ ${ }^{1}$ Rheumatology Unit, L. Sacco University Hospital of Milan, Italy; \\ ${ }^{2}$ Dipartimento di Medicina Interna e Geriatria, Università di Palermo, Italy; \\ ${ }^{3}$ Rheumatology Unit, Nuovo Ospedale S. Giovanni di Dio, ASL 10, Florence, Italy; \\ ${ }^{4}$ Department of Internal Medicine and Medical Specialities, Sapienza University of Rome, Italy; \\ ${ }^{5}$ Department of Clinical Neurophysiology \& Pain Rehabilitation Unit, Rehabilitation Institute of Montescano, \\ IRCCS, Foundation Salvatore Maugeri, Pavia Italy; \\ 'Hermanas Hospitalarias, FoRiPsi, Department of Clinical Neurosciences, \\ Villa San Benedetto Menni, Albese con Cassano, Como, Italy
}

\section{SUMMARY}

Fibromyalgia (FM) is a chronic pain syndrome that affects at least $2 \%$ of the adult population. It is characterised by widespread pain, fatigue, sleep alterations and distress, and emerging evidence suggests a central nervous system (CNS) malfunction that increases pain transmission and perception. FM is often associated with other diseases that act as confounding and aggravating factors, such as rheumatoid arthritis (RA), spondyloarthritides $(\mathrm{SpA})$, osteoarthritis (OA) and thyroid disease. Mechanism-based FM management should consider both peripheral and central pain, including effects due to cerebral input and that come from the descending inhibitory pathways. Rheumatologists should be able to distinguish primary and secondary FM, and need new guidelines and instruments to avoid making mistakes, bearing in mind that the diffuse pain of arthritides compromises the patients' quality of life.

Key words: Arthritides; pain; fibromyalgia; rheumatic diseases.

Reumatismo, 2012; 64 (4): 286-292

\section{INTRODUCTION}

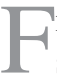
ibromyalgia (FM) is a chronic pain syndrome that affects at least $2 \%$ of the adult population, and is characterised by widespread pain, fatigue, sleep alterations, and distress (1). It is currently defined as chronic widespread pain (CWP) with allodynia or hyperalgesia to pressure pain, and classified as one of the large group of softtissue pain syndromes (1-3). There is growing evidence that it may involve a central nervous system (CNS) malfunction that increases pain transmission and perception (4). The widespread nature of spontaneous pain in FM involves general mechanisms that may include the spinal or supraspinal modulation of normal peripheral input, or effector mechanisms that alter sensitivity to peripheral pain $(4,5)$. The etiology of FM is not completely understood and the syndrome is influenced by factors such as stress, medical illness, and a variety of pain conditions including trauma, infections (e.g., hepatitis $\mathrm{C}$ virus, HIV and Lyme disease), emotional stress, catastrophes (e.g., war), autoimmune diseases and other pain conditions (6-8). FM is often associated with other diseases that act as confounding and aggravating factors, such as rheumatoid arthritis (RA), spondyloarthritides ( $\mathrm{SpA})$, osteoarthritis (OA) and thyroid disease (8).

The aim of this review is to analyse the impact of FM in arthritides and their treatment.

\section{Pain as a symptom of FM and other rheu- matic diseases}

The most frequent and typical symptoms of FM are generalised pain, stiffness, fatigue, and poor sleep (9), but the defining symptom is widespread chronic pain lasting at least three months, usually in all four limbs and the upper or lower back. The pain has been described in many ways (burning, searing, tingling, shooting, stabbing, deeply aching, sharp, or "feeling bruised all 
over") and, like stiffness, it is often worsened by cold or damp weather, anxiety or stress, excessive or too little physical activity, poor sleep, and noise $(1,9)$. About $65 \%$ of the patients say they "hurt all over", and this may be useful in differentiating FM from other conditions $(8,9)$.

However, musculoskeletal pain is common in patients with rheumatic diseases, and it is not always easy to discover its cause. Pain is certainly the cornerstone of a diagnosis of FM, but it is accompanied an array of multi-system symptoms that are common to various musculoskeletal conditions (such as fatigue, Raynaud's phenomenon, sleep disturbances, muscle stiffness, sicca syndrome, irritable bowel disease, depression and anxiety), and the absence of specific laboratory findings can easily lead to a misdiagnosis $(9,10)$. As FM-like symptoms are frequently encountered, differential diagnoses against other causes of chronic pain are essential (8). When the pain involves a large number of joints, it may be confused with the widespread pain of FM, but the degree of pain measured by means of a visual analogue scale (VAS) is not helpful in distinguishing FM from conditions such as RA or OA $(11,12)$. Furthermore, as it can co-exist with other immuno-inflammatory diseases, many rheumatic and non-rheumatic diseases can easily be misdiagnosed as FM (12). There are no instrumental tests to confirm the diagnosis, but many of the differential diagnoses can be excluded by means of an extensive clinical examination and patient history.

One study (13) of inaccurate diagnoses of FM in a cohort of patients referred to a rheumatology clinic found that FM was confirmed in only $34 \%$ of the patients presenting with musculoskeletal pain. The symptoms distinguishing FM and non-FM patients were tender points (TPs) $(\mathrm{P}<0.0001)$ and fatigue $(\mathrm{P}=0.0003)$, whereas prolonged early morning stiffness clinically distinguished non-FM patients, although it was also found in $25 \%$ of the FM patients. Given the large number of misdiagnoses $(66 \%)$, the authors concluded that a wider range of diseases should be considered when making a differential diagnosis.
Di Franco et al. (14) studied 427 outpatients, 57 of whom had been previously misdiagnosed as having other musculoskeletal disorders (the MSD group); the others had been previously correctly diagnosed as having FM or were diagnosed as having it during the course of the study. The FM and MSD groups were comparable in terms of demographic data and referral patterns, but the patients with MSD had significantly more frequent antinuclear antibodies (ANA) and low titres of rheumatoid factor (RF) positivity. Moreover, there were significant differences in the use of non-steroidal anti-inflammatory drugs (NSAIDs), steroids, DMARDs and biological agents $(\mathrm{P}<0.0001$ for each class of drugs) between the two groups (14). The authors concluded that, as FM symptoms generally respond poorly to various treatments, the consequences of a misdiagnosis and mistreatment are a later diagnosis and a longer disease duration, which can worsen the patients' quality of life and increase the economic burden on society.

Many differential diagnoses can be excluded by an extensive clinical examination and patient history but, given the considering overlap of FM with other medical conditions, treating physicians need to remain alert. Chest X-ray and abdominal ultrasonography are the first steps in the general assessment of all the patients with suspected FM (10), but establishing a diagnosis may be difficult because of the multifaceted nature of the syndrome.

\section{Fibromyalgia and rheumatoid arthritis}

FM may occur alone (primary FM) or in combination with other diseases (secondary FM): $25 \%$ of FM patients have RA, and a recent study estimated that the incidence of new-onset FM in RA patients was $20 \%$ (15). In the same study, $7.4 \%$ of the RA patients satisfied the criteria for FM at the time of the last observation, and $19.8 \%$ satisfied them at some time during the followup, with a similar incidence in men $(7.0 \%)$ and women $(8.1 \%)$. The factors that correlated with developing FM in RA included social disadvantage, psychological distress, comorbidities, and the severity of the RA. 
RA is a complex inflammatory arthritis whose activity cannot be assessed using a single physical or laboratory measure (16). In 1993, the Disease Activity Score (DAS) was developed to provide a quantifiable composite measure of RA disease activity (16-18). It was based on a combination of swollen and tender joint counts, the acutephase response and general health, and the use of statistical measures that included discriminant and multiple regression analysis. It has been found that the has more power than other indices or single variables in discriminating low from high levels of disease activity, and the area under the curve of Creactive protein (CRP) correlates well with joint damage over time (19).

DAS28 is a modified version of the original DAS that is based on the same four variables, but uses swollen and tender joint counts of only 28 joints: it is a continuous index ranging from 0 to 9.4 , in which low disease activity is defined as a score of $\leq 3.2$, moderate disease activity is defined as a score of $>3.2$ and $\leq 5.1$, and high disease activity is defined as a score of $>5.1$ (20). A commonly used cut-off point for remission is $<2.6(17,18,20)$. During the current decade, rheumatologists have witnessed a series of major developments in the treatment of RA, including new treatments that make it possible to aim at stringent targets, including remission. Outcome assessments have also been improved and become easier to use in clinical practice, thus allowing the assessment of disease activity and response to treatment, and the categorisation of disease activity states.

Patients with RA should undergo a careful clinical examination in order to evaluate the clinical association with FM, optimise therapy, reduce healthcare costs. Pain, fatigue and stiffness are common in RA, and FM plays a major role $(9,16)$. Wolfe and Michaud (21) found that $17 \%$ of their RA patients had FM, and that their RA was more subjectively and objectively severe. Another very interesting study found that patients with RA and concomitant FM had significantly greater disease activity as measured by DAS28, but the patients with RA alone showed significantly more severe joint destruction. One study on 120 RA patients (including 25 with concomitant FM) found significantly higher tender joint counts (TJC) and global health VAS values, the subjective measurements that contribute most to the differences in disease activity in RA patients with and without FM; however, the doctor's global heath VAS values incorporated in a simple disease activity index (SDAI) and a clinical disease activity index (CDAI) were also markedly higher in the patients with both diseases (22). Composite indices such as DAS28, SDAI and CDAI are widely used in everyday clinical practice and clinical trials, but they may be insufficient to evaluate real inflammatory activity in cases of RA associated with a chronic pain syndrome such as FM; rheumatologists need to be aware of these limitations.

Ultrasonography (US) can identify the elements characteristic of RA or FM, and therefore help clinicians to make a correct diagnosis (23). An examination performed by an expert US rheumatologist using the newer colour Doppler US and the spectral Doppler Resistivity Index, can differentiate inflammatory and non-inflammatory disease and be used as an indicator of RA. Meenagh et al. (24) have shown that the majority of FM patients have no significant pathological US abnormalities.

\section{Fibromyalgia and spondyloathropathies} Spondyloarthropathies (SpA) are a group of inflammatory arthritides consisting of ankylosing spondylitis (AS), reactive arthritis, arthritis/spondylitis associated with psoriasis (PsA), and arthritis/spondylitis associated with inflammatory bowel diseases, all of which have sometimes been found to be associated with FM.

\section{Fibromyalgia and psoriatic arthritis}

Psoriatic arthritis (PsA) is a chronic inflammatory arthropathy of unknown etiology that is associated with psoriasis. It occurs in up to one-third of patients with psoriasis and has a heterogeneous pattern expressed by various manifestations including mono-oligoarthritis (an erosive and destructive polyarthritis indistinguish- 
able from RA) and spondyloarthropathy with axial involvement or enthesitis (25). The prevalence of FM in PsA patients is unknown, although one study found tenderness in ten or more fibrotic sites in 24\% of patients with PsA and $57 \%$ of RA patients (26). If there are no objective signs of inflammation at entheseal sites, it can be difficult to distinguish enthesitic and fibromyalgic pain clinically because the symptoms and signs may be aspecific and relatively indistinguishable from those of FM. In a cross-sectional study involving 266 patients with PsA and 120 patients with FM by Marchesoni et al. (26), univariate analysis showed that the latter had higher mean tender point and enthesitis scores, more somatic symptoms, and responded less to NSAIDs. Multivariate analysis showed that the presence of $\geq 6$ FM-associated symptoms and $\geq 8$ tender points was the best predictor of FM. The authors concluded that PsA and FM share clinical features, and that the number of FM-associated symptoms and tender point count were the most useful variables for discriminating FM.

As in patients with RA, US permits the early detection and characterisation of the inflammatory process in different tissues in PsA patients, and helps in the differential diagnosis.

\section{Fibromyalgia and ankylosing spondylitis}

Ankylosing spondylitis (AS) is a chronic inflammatory disease that affects the axial skeletal system, causing pain and functional incapacity $(28,29)$. To measure the impact of AS on a patient's life, questionnaires are used to assess disease activity (BASDAI), functional incapacity (BASFI), and the quality of life (ASQoL). The impact of FM can be assessed using the Fibromyalgia Impact Questionnaire (FIQ) (30), but only some studies have demonstrated correlations between FM and AS.

Aloush et al. (31) studied 36 patients with AS (18 males and 18 females) and diagnosed FM in $50 \%$ of the women but none of the men. They pointed out that patients with two concomitant diseases were more functionally impaired, which led to higher BASDAI and BASFI scores than those of patients with only one disease. In another study of 71 patients, the prevalence of FM was $15 \%$ (45.5\% males and $54.5 \%$ females): these patients had significantly higher BASDAI scores $(\mathrm{P}<0.001$ at Student's $t$-test and $\mathrm{P}=0.002940$ at Wilcoxon's test), BASFI scores $(\mathrm{P}<0.001$ at Student's $t$-test and $\mathrm{P}=0.001992$ at Wilcoxon's test), and ASQoL scores $(\mathrm{P}=0.0019$ at Student's $t$-test and $\mathrm{P}=0.001884$ at Wilcoxon's test) (32). A cross-sectional Spanish study of 462 patients with definite AS found that the prevalence of FM was $4.11 \%$ in the population as a whole, but $10.83 \%$ among the women (33). The BASDAI, BASFI and total Bath AS radiology index (BASRI) were strongly influenced by the presence of FM and, when the authors used the inverse relationship between BASDAI or BASFI and total BASRI to make a ratio, they found that the patients with a BASDAI/BASRI ratio of $\geq 1.5$ or a BASFI/BASRI ratio of $\geq 1.08$ were very likely to have FM. They concluded that FM distorts the measures of activity and functional damage in patients with AS, and that the BASDAI/BASRI and BASFI/BASRI ratios are a useful means of identifying such patients and avoiding over-treatment (33).

The diagnosis of AS is based on the presence of chronic vertebral pain associated with morning stiffness, both of which are frequent in FM patients (29). Anxiety, depression, fatigue, and sleep disorders, all of which are closely associated with pain, are significant in FM and very frequently reported by patients with AS.

The current indication for the use of biological agents in AS is generally based on the activity index but, before biological agents are prescribed, we suggest that the possible presence of concomitant FM should be investigated and the positive patients should be adequately treated and reassessed. AS can also easily be mistaken for FM because both occur in young patients who may have constitutional symptoms such as malaise, fatigue and impaired sleep (34). However, the hallmark of AS is sacroiliitis, which can be identified early by means of magnetic resonance imaging (MRI) (35) and is not found in patients with FM. 


\section{Fibromyalgia and osteoarthritis}

Patients with OA and FM have more problems sleeping $(70 \%)$ that correlate with fatigue (36), and are also associated with more severe OA and depression.

Bican et al. (37) compared 59 OA patients (90 knees) with FM who underwent primary total knee arthroplasty (TKA) with FMfree control patients undergoing the same surgery. The FM patients had lower preand postoperative SF-36 scores and, after 3.4 years of follow-up, were less satisfied with their TKA. However, their post-surgical improvement was comparable with that of the controls. FM should not be considered a contraindication for surgery.

\section{The implications of concomitant FM in chronic diseases}

The multiple and complex mechanisms underlying FM (38) include some that involve temporal summation (wind-up), long-term potentiation (LTP), heterosynaptic potentiation, dysfunctional descending pain inhibition, and activation of the descending facilitatory pathway (38). Although pain in RA is often thought of as inflammatory and peripheral (4), central pain may also be important. Patients with RA have general hyperalgesia to mechanical and thermal stimuli in various body areas, not just at the sites of their inflamed joints (39). They also have enhanced pain reactivity as reflected by serum TNF levels. Wendler et al. (40) used electroencephalography to show that, in comparison with age- and gendermatched controls, RA patients have greater cortical responses to repeated noxious stimulation, thus suggesting changes in the CNS modulation of pain. Moreover, it has been shown that the relationships between inflammation, psychosocial factors, and peripheral and central pain processing are intricately inter-related not only in patients with RA (41), but also in those with OA. One small study has suggested that OA patients also have widespread hyperalgesia to mechanical or heat stimuli (42), and Kosek and Ordeberg found that patients with hip OA showed reduced descending analgesic activity, which partially normalised after hip arthroplasty, thus suggesting that the central factors were at least partially being driven by peripheral pain factors (43). Furthermore, randomised controlled trials have demonstrated that compounds that alter pain neurotransmitters centrally, such as serotonin and norepinephrine (eg, duloxetine, tricyclic antidepressants), are efficacious in OA (44).

Given these findings, the most important implication of concomitant FM in patients with chronic structural diseases is to recognise its presence in order to ensure optimal management (45). For example, FM symptoms with multiple tender points in patients with RA should not be automatically attributed to increased RA activity, and the patients should not be prescribed higher doses of a biological agent or corticosteroids without an appropriate TP examination and laboratory evaluation (45). Attention should be given to managing the FM by means of centrally acting medications, cognitive behavioural therapy, and sleep management. An OA patient with severe pain will require analgesics that act both peripherally and centrally, such as cyclobenzaprine, pregabalin, duloxetine, or milnacipran (45).

A number of different pharmacological treatments, including antidepressants, NSAIDS, opioids, sedatives, muscle relaxants and anti-epileptic agents, have been used to treat FM with varying results (46). Moreover, physical exercise and multimodal cognitive behavioural therapy seem to be the most widely accepted and beneficial forms of non-pharmacological therapy (47). Given that no single treatment for pain is ideal, a multimodal approach is recommended.

\section{CONCLUSIONS}

The presence of diffuse pain in arthritides does not increase overall mortality but compromises the patients' quality of life. Mechanism-based management should consider both peripheral and central pain, including the effects due to cerebral input and from the descending inhibitory pathways. Optimal therapy should take into 
account symptoms such as fatigue, mood, sleep, and the overall quality of life. Rheumatologists should be able to recognise and distinguish primary and secondary FM, and need new guidelines and instruments to avoid making mistakes.

\section{REFERENCES}

1. Cazzola M, Sarzi Puttini P, Stisi S, et al. (Italian Fibromyalgia Network). Fibromyalgia syndrome: definition and diagnostic aspects. Reumatismo. 2008; 60 (Suppl. 1): 3-14.

2. Nampiaparampil DE, Shmerling RH. A review of fibromyalgia. Am J Manag Care. 2004; 10: 794-800.

3. Ablin JN, Buskila D, Van Houdenhove B, et al. Is fibromyalgia a discrete entity? Autoimmun Rev. 2012; 11: 585-8.

4. Sarzi-Puttini P, Atzeni F, Mease PJ. Chronic widespread pain: from peripheral to central evolution. Best Pract Res Clin Rheumatol. 2011; 25: 133-9.

5. Staud R. The abnormal central pain processing mechanism in patients with fibromyalgia. Fibromyalgia Frontiers. 2002; 10: 18.

6. Buskila D, Atzeni F, Sarzi-Puttini P. Etiology of fibromyalgia: the possible role of infection and vaccination. Autoimmun Rev. 2008; 8: 41-3.

7. Buskila D, Sarzi-Puttini P. Fibromyalgia and autoimmune diseases: the pain behind autoimmunity. Isr Med Assoc J. 2008; 10: 77-8.

8. Atzeni F, Cazzola M, Benucci M, et al. Chronic widespread pain in the spectrum of rheumatological diseases. Best Pract Res Clin Rheumatol. 2011; 25: 165-71.

9. Cassisi G, Sarzi-Puttini P, Alciati A, Casale R, Bazzichi L, Carignola R, et al. Symptoms and signs in fibromyalgia syndrome. Reumatismo. 2008; 60 (Suppl. 1): 15-24.

10. Atzeni F, Salaffi F, Bazzichi L, et al. Italian Fibromyalgia Network. The evaluation of the fibromyalgia patients. Reumatismo. 2008; 60 (Suppl.1): 36-49.

11. Bliddal H, Danneskiold-Samsøe B. Chronic widespread pain in the spectrum of rheumatological diseases. Best Pract Res Clin Rheumatol. 2007; 21: 391-402.

12. Yunus MB. Central sensitivity syndromes: a new paradigm and group nosology for fibromyalgia and overlapping conditions, and the related issue of disease versus illness. Semin Arthritis Rheum. 2008; 37: 339-52.

13. Fitzcharles MA, Boulos P. Inaccuracy in the diagnosis of fibromyalgia syndrome: analysis of referrals. Rheumatology. 2003; 42: 263-7.

14. Di Franco M, Iannuccelli C, Bazzichi L, et al. Misdiagnosis in fibromyalgia: a multicentre study. Clin Exp Rheumatol. 2011; 29: S104-8.
15. Wolfe F, Hauser W, Hassett AL, et al. The development of fibromyalgia - I: examination of rates and predictors in patients with rheumatoid arthritis (RA). Pain. 2011; 152: 249-50.

16. Ferraccioli G, Gremese E. Pathogenetic, clinical and pharmaco-economic assessment in rheumatoid arthritis (RA). Intern Emerg Med. 2011; 6 (Suppl. 1): 11-5.

17. Burmester GR. RA in 2011: advances in diagnosis, treatment and definition of remission. Nat Rev Rheumatol. 2012; 8: 65-6.

18. Dougados M, Ripert M, Hilliquin P, et al. The influence of the definition of patient global assessment in assessment of disease activity according to the Disease Activity Score (DAS28) in rheumatoid arthritis. J Rheumatol. 2011; 38: 2326-8.

19. van der Heijde DM, van't Hof MA, van Riel PL, et al. Judging disease activity in clinical practice in rheumatoid arthritis: first step in the development of a disease activity score. Ann Rheum Dis. 1990; 49: 916-20.

20. Landewé $R$, van der Heijde D, van der Linden S, Boers M. Twenty-eight-joint counts invalidate the DAS28 remission definition owing to the omission of the lower extremity joints: a comparison with the original DAS remission. Ann Rheum Dis 2006; 65: 637-41.

21. Wolfe F, Michaud K. Severe rheumatoid arthritis (RA), worse outcomes, comorbid illness, and sociodemographic disadvantage characterize RA patients with fibromyalgia. J Rheumatol. 2004; 31: 695-700.

22. Toms J, Soukup T, Bradna P, Hrncir Z. Disease activity composite indices in patients with rheumatoid arthritis and concomitant fibromyalgia. J Rheumatol. 2010; 37: 468.

23. Grassi W, Salaffi F, Filippucci E. Ultrasound in rheumatology. Best Pract Res Clin Rheumatol. 2005; 19: 467-85.

24. Meenagh G, Sakellariou G, Iagnocco A, et al. Ultrasound imaging for the rheumatologist XXXIX. Sonographic assessment of the hip in fibromyalgia patients. Clin Exp Rheumatol. 2012; 30: 319-21.

25. Atzeni F, Ventura D, Batticciotto A, et al. Interleukin 6 blockade: tocilizumab in psoriatic arthritis. J Rheumatol 2012; 89: 97-9.

26. Buskila D, Langevitz P, Gladman DD, et al. Patients with rheumatoid arthritis are more tender than those with psoriatic arthritis. J Rheumatol. 1992; 19: 1115-9.

27. Marchesoni A, Atzeni F, Spadaro A, et al. Identification of the clinical features distinguishing psoriatic arthritis and fibromyalgia. J Rheumatol. 2012; 39: 849-55.

28. Yunus MB. The prevalence of fibromyalgia in other chronic pain conditions. Pain Res Treat. 2012; 2012: 584573.

29. Sieper J. How to define remission in ankylosing spondylitis? Ann Rheum Dis. 2012; 71 (Suppl. 2): i93-5. 
30. Sarzi-Puttini P, Atzeni F, Fiorini T, et al. Validation of an Italian version of the Fibromyalgia Impact Questionnaire (FIQ-I). Clin Exp Rheumatol. 2003; 21: 459-64.

31. Aloush V, Ablin J, Reitblat T, et al. Fibromyalgia in women with ankylosing spondylitis. Rheumatol Intern. 2007; 27: 865-8.

32. Azevedo VF, Paiva Edos S, Felippe LR, Moreira RA. Occurrence of fibromyalgia in patients with ankylosing spondylitis. Rev Bras Reumatol 2010; 50: 646-50.

33. Almodóvar R, Carmona L, Zarco P, et al. Fibromyalgia in patients with ankylosing spondylitis: prevalence and utility of the measures of activity, function and radiological damage. Clin Exp Rheumatol. 2010; 28: S33-9.

34. Khan MA. Update on spondyloarthropathies. Ann Intern Med. 2002; 136: 896-907.

35. Amrami KK. Imaging of the seronegative spondyloarthopathies. Radiol Clin North Am. 2012; 50: 841-54.

36. Hawker GA, French MR, Waugh EJ, et al. The multidimensionality of sleep quality and its relationship to fatigue in older adults with painful osteoarthritis. Osteoarthritis Cartilage. 2010; 18: 1365-71.

37. Bican O, Jacovides C, Pulido L, et al. Total knee arthroplasty in patients with fibromyalgia. J Knee Surg. 2011; 24: 265-71.

38. Woolf CJ. Central sensitization: implications for the diagnosis and treatment of pain. Pain. 2010; 152: S2-S15.

39. Edwards RR, Wasan AD, Bingham CO, et al. Enhanced reactivity to pain in patients with rheumatoid arthritis. Arthritis Res Ther. 2009; 11: 114-23.
40. Wendler J, Hummel T, Reissinger M, et al. Patients with rheumatoid arthritis adapt differently to repetitive painful stimuli compared to healthy controls. J Clin Neurosci. 2001; 8: 272-7.

41. Lee YC, Chibnik LB, Wasan AD, et al. The relationship between disease activity, sleep, psychiatric distress and pain sensitivity in rheumatoid arthritis: a cross-sectional study. Arthritis Res Ther. 2009; 11: R160.

42. Graven-Nielsen T, Arendt-Nielsen L. Assessment of mechanisms in localized and widespread musculoskeletal pain. Nat Rev Rheumatol. 2010; 6: 599-606.

43. Kosek E, Ordeberg G. Abnormalities of somatosensory perception in patients with painful osteoarthritis normalize following successful treatment. Eur J Pain. 2000; 4: 229-38.

44. Chappell AS, Desaiah D, Liu-Seifert H, et al. A double-blind, randomized, placebo-controlled study of the efficacy and safety of duloxetine for the treatment of chronic pain due to osteoarthritis of the knee. Pain Pract. 2011; 11: 33-41.

45. Goldenberg DL, Clauw DJ, Fitzcharles MA. New concepts in pain research and pain management of the rheumatic diseases. Semin Arthritis Rheum. 2011; 41: 319-34.

46. Sarzi-Puttini P, Buskila D, Carrabba M, et al. Treatment strategy in fibromyalgia syndrome: where are we now? Semin Arthritis Rheum. 2008; 37: 353-65.

47. Sarzi-Puttini P, Atzeni F, Salaffi F, et al. Multidisciplinary approach to fibromyalgia: what is the teaching? Best Pract Res Clin Rheumatol. 2011; 25: 311-9. 